\title{
Lidil
}

Revue de linguistique et de didactique des langues

$43 \mid 2011$

Le rapport au savoir dans les discours professionnels

\section{Discours de formateurs et rapport au savoir : l'explicitation située de l'action comme discours professionnel}

Trainers' discourse and knowledge construction: situated reflexivity as professional discourse

\section{Laurent Filliettaz}

\section{OpenEdition}

Journals

Édition électronique

URL : http://journals.openedition.org/lidil/3101

DOI : 10.4000/lidil.3101

ISSN : $1960-6052$

Éditeur

UGA Éditions/Université Grenoble Alpes

Édition imprimée

Date de publication : 30 mai 2011

Pagination : 11-25

ISBN : 978-2-84310-201-1

ISSN : $1146-6480$

\section{Référence électronique}

Laurent Filliettaz, «Discours de formateurs et rapport au savoir : l'explicitation située de l'action comme discours professionnel », Lidil [En ligne], 43 | 2011, mis en ligne le 30 novembre 2012, consulté le 19 avril 2019. URL : http://journals.openedition.org/lidil/3101 ; DOI : 10.4000/lidil.3101 


\title{
Discours de formateurs et rapport au savoir : I'explicitation située de l'action comme discours professionnel
}

\author{
Laurent Filliettaz*
}

\begin{abstract}
RÉSUMÉ
Dans les démarches d'observation de la pratique telles qu'elles sont conduites par le chercheur dans son rapport avec les professionnels, des formes spontanées et situées d'explicitation sont parfois proposées par les praticiens, en rapport avec les activités en cours d'accomplissement. Ces formes spontanées de l'explicitation nous intéressent en ce qu'elles rendent visibles des rapports aux savoirs issus de la pratique. Dans cet article, des données empiriques consistant en des échanges spontanés entre chercheurs et formateurs dans le champ de la mécanique permettent de mettre en évidence la diversité des savoirs mobilisés par les formateurs et la manière dont ceux-ci se positionnent à l'égard d'une professionnalité à la fois technique et didactique.
\end{abstract}

\section{ABSTRACT}

When practitioners meet with researchers in the natural conditions of their ordinary activities, they sometimes spontaneously comment and reflect on various dimensions of their professional practice. This paper proposes to explore the methodological potentialities of these accounts so as to illuminate the relations between research and practice in the context of vocational education. To do so, the paper refers to a research program currently conducted on teaching and learning practices within the Swiss dual VET system. This program provides empirical evidence regarding the ways teachers or trainers interact with researchers during ordinary training activities. The paper proposes an empirical approach to these sequences of interaction and uses these data to illustrate the diverse vocational knowledge trainers are able to reflect on when making their actions accountable to researchers in such contexts.

* Université de Genève. 


\section{La mise en discours des savoirs professionnels}

L'abondante littérature publiée ces dernières années dans le champ de l'analyse de la pratique en formation (voir Marcel et al., 2002; Relieu et al., 2004; Plazaola Giger et Stroumza, 2007) opère souvent, et non sans (bonnes) raisons, une distinction méthodologique entre l'observation des pratiques et l'étude des discours sur les pratiques. Si la première démarche revient généralement pour le chercheur à prêter attention aux comportements des acteurs en situation et à centrer les observations sur les dynamiques de l'action «située», la seconde s'applique à aménager des espaces discursifs et des temporalités spécifiques permettant un retour réflexif des acteurs sur leur activité. Des techniques d'entretien diverses et aujourd'hui abondamment pratiquées dans la recherche et la formation (l'entretien d'explicitation, l'instruction au sosie, l'entretien en auto-confrontation, etc.) relèvent clairement de cette seconde orientation.

L'objectif de cette contribution est de remettre en question le caractère étanche de cette distinction et d'explorer quelques-unes des réalités discursives qui se déploient parfois à la marge des démarches ainsi opposées. En particulier, il s'agira de constater que, dans les démarches d'observation de la pratique telles qu'elles sont conduites par le chercheur dans son rapport avec les professionnels en activité, des formes spontanées et situées d'explicitation sont parfois proposées par les praticiens, en rapport avec les activités en cours d'accomplissement. Ce sont ces formes langagières qui seront désignées ici comme des «explicitations situées » et que nous proposons de considérer comme des formes particulières de discours professionnels.

Ces formes situées de l'explicitation nous intéressent en ce qu'elles apportent un éclairage à propos de deux ordres de questionnements qui nous apparaissent comme des structurants majeurs de la recherche sur les processus de professionnalisation. Le premier ordre de questionnement est de nature épistémologique et a trait à la nature des savoirs qui fondent une pratique professionnelle, que celle-ci relève de l'enseignement en milieu scolaire ou de toute autre profession. Le second ordre de questionnement est de nature davantage méthodologique et concerne les conditions auxquelles ces savoirs peuvent être rendus visibles dans les discours produits par les professionnels eux-mêmes. De ce point de vue, cet article visera à éprouver empiriquement la valeur heuristique de ces explicitations situées de l'action en contribuant à éclairer les questions suivantes : quelles formes d'interprétation de leur agir les 
professionnels peuvent-ils produire en cours d'accomplissement de leur activité? Quelles dimensions de l'activité ressortent de leurs discours et quelles sortes de savoirs sont rendues visibles à cette occasion? Les formes situées de l'explicitation de l'action viennent-elles faciliter l'accès aux savoirs sous-jacents à la pratique professionnelle? Quelles en sont les potentialités propres et les limites, pour une compréhension accrue des processus de professionnalisation?

Pour esquisser des éléments de réponse à ces questions, nous centrerons notre propos sur le champ de la formation professionnelle initiale portant sur des métiers techniques. Ce ne sont donc pas des discours d'enseignants en formation qui feront l'objet de notre analyse, mais des discours de formateurs d'apprentis, adressés à des chercheurs, et recueillis dans le déroulement même des activités de formation accomplies et observées. Ces réflexions s'adossent à un programme de recherche que nous conduisons depuis 2005 dans le champ de la formation professionnelle initiale en Suisse et dans lequel nous cherchons notamment à mieux connaitre les éléments de compétence sur lesquels s'organise l'activité des enseignants et des formateurs de la formation professionnelle (Filliettaz, de Saint-Georges et Duc, 2008, 2009, 2010). À cette fin, des méthodes ethnographiques d'orientation interactionnelle ont été mobilisées dans le but de mieux comprendre comment les formateurs mettent en circulation des savoirs à l'occasion d'interactions impliquant des apprentis. Dans un important corpus d'enregistrements audio-vidéo documentant des activités de formation authentiques accomplies en situation de formation et de travail, nous avons également entrepris récemment de repérer et d'étudier des séquences spécifiques durant lesquelles, en cours d'enregistrement, les formateurs s'adressent spontanément au chercheur-observateur dans le but de commenter certaines des propriétés des activités en cours d'accomplissement. Dans cet article, nous présentons, sous la forme d'une étude de cas, quelques-unes des séquences d'interaction ainsi recueillies avec un double objectif : celui d'abord de mettre en évidence des éléments de savoir qui sous-tendent l'activité des formateurs et tels qu'ils sont portés à l'attention du chercheur à l'occasion de ces explicitations spontanées; celui ensuite de réfléchir aux implications identitaires d'une telle explicitation de ces savoirs pour les formateurs eux-mêmes. Nous pensons ainsi pouvoir éclairer quelque peu la nature des rapports qui se tissent, chez ces formateurs, entre leur pratique professionnelle et les savoirs qui les sous-tendent. 


\section{Explicitation située et rapports aux savoirs en formation professionnelle initiale}

La formation professionnelle initiale telle qu'elle est organisée en Suisse prend généralement place dans une pluralité de lieux et d'institutions de formation : des écoles professionnelles, des places de travail en entreprise et des centres de formation (Dubs, 2006). Les apprentis y côtoient une vaste palette de professionnels, dont certains sont des enseignants professionnels et d'autres des travailleurs expérimentés endossant, occasionnellement ou sur une base régulière, une responsabilité d'encadrement ou de formation. C'est à cette seconde catégorie de formateurs qu'appartient le professionnel dont nous étudierons le discours dans les extraits ci-dessous.

Ces extraits ont été enregistrés dans le contexte d'un centre de formation professionnelle d'une entreprise publique du canton de Genève, dont les apprentis engagés dans une formation technique disposent d'une initiation d'une durée de quatre mois, destinée à leur permettre de construire des aptitudes techniques de base avant d'effectuer divers stages au sein de l'entreprise. L'atelier dont il est question ici porte sur la mécanique générale. Il est animé et encadré par un employé, qui endosse ici ponctuellement un rôle de formateur, mais qui ne dispose pas d'une formation pédagogique particulière le préparant à ces tâches. Dans cet atelier de mécanique générale, les jeunes en formation apprennent à maitriser des techniques de base en lien avec la transformation du métal : le limage, le traçage, le perçage, le taraudage, etc. Après une initiation de sept semaines, le formateur en charge de cet atelier soumet les apprentis à une tâche inhabituelle, dont la consigne est de réaliser une pièce métallique selon un plan, et ce sans explication supplémentaire et sous contrainte de temps. Les apprentis ont une journée entière pour fabriquer cette pièce, qui combine l'ensemble des techniques apprises à ce jour (voir le produit final dans l'illustration 1 ci-dessous). Outre la réalisation de chanfreins, de trous et d'inscriptions, cette tâche comporte également une difficulté supplémentaire que les apprentis n'ont encore jamais rencontrée et pour laquelle ils doivent faire preuve de créativité. La difficulté consiste à réaliser une encoche arrondie dans la partie inférieure droite de la pièce.

Le premier extrait prend place peu de temps après la distribution de la consigne. Il est encore tôt le matin, et les apprentis s'engagent rapidement dans la tâche de sorte à ne pas perdre de temps (voir l'image 2). Alors que les apprentis commencent par limer leur pièce pour la mettre 
à la cote, le formateur (FOR) s'approche du chercheur (CHE) et propose le commentaire suivant de l'activité en cours de réalisation.

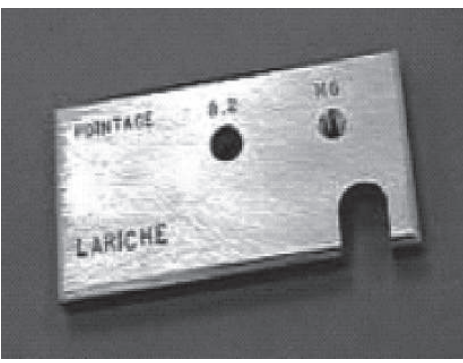

Image 1: Illustration du produit final de l'exercice.

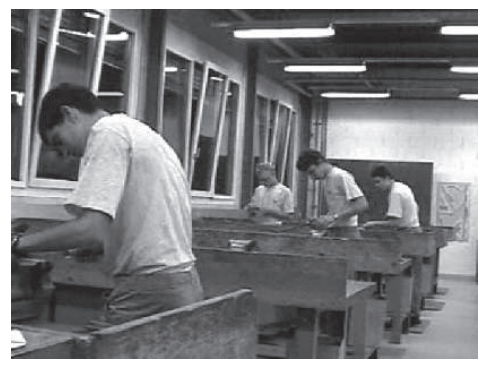

Image 2 : Disposition des apprentis dans l'atelier.

Extrait 1. - Pièce métallique (Film no 10, $04^{\prime} 21-06^{\prime} 20$ ).

04'21 1. FOR : alors là aujourd'hui au fait ils vont faire beaucoup d'erreurs $<$ mhmm > vu qu'ils sont un petit peu stressés/ vu qu'ils ont un temps qui est donné/ et on va voir ceux qui ont qui vont passer par dessus/ puis ceux quil.

2. CHE : où ça va bloquer quoil

3. FOR : qui vont bloquer ouais $\backslash$. alors on peut déjà voir déjà certains qui: qui: qui sont pas corrects/ . qui sont pas en bonne position/ ...

04'58 4. CHE : ils ont l'habitude de commencer par mettre les/ les longueurs à l'équerre/

5. FOR : alors il faut mettre d'équerre la première face/ après on met d'équerre la deuxième face et deux longueurs en fait $\backslash$...

05’26 6. donc là on voit qu'ils sont tous sous pression parce qu'il y a des pièces qui tombent/ des positions qui sont pas justes/ puis en fait y a pas de réflexion avant de commencer la pièce $\backslash<$ ouais> qu'est-ce qu'il faut que je fasse/ pourquoi comment/

7. CHE : ils foncent tous tout de suite dans la

8. FOR : ouais ils foncent dessus sans se poser la question euh comment on va la faire en fait $\backslash$..

9. puis on verra certains d'ici cinq dix minutes ils vont se dire ah/ non il faut que je modifie il faut que je fasse comme ça comme ça comme çal..

10. puis d'autres qui vont y voir quand ce sera trop tard $\backslash$

11. CHE : mhmm\. si jamais ils doivent recommencer ils n'arriveront pas à la finirl

06 '20 12. FOR : voilàl 
Cette séquence d'interaction est spontanément initiée par le formateur («alors là aujourd'hui au fait ils vont faire beaucoup d'erreurs » [1.1]). Elle met en lumière certains savoirs sous-jacents à la situation et qui portent sur différentes dimensions de cette activité collective.

Ce sont d'abord des savoirs à caractère technique en lien avec l'activité en cours d'accomplissement qui sont rendus visibles dans le discours de ce formateur («alors il faut mettre d'équerre la première face/ après on met d'équerre la deuxième face et deux longueurs en fait» [1.5]). À cette occasion, le formateur porte à l'attention du chercheur l'organisation processuelle de la tâche et la séquence d'opérations que les apprentis doivent suivre pour la réaliser.

Cette séquence permet ensuite de mettre en évidence une forme de capacité diagnostique du formateur dans la situation, centrée en particulier sur les comportements des apprentis et leur engagement dans la tâche. Le formateur mentionne premièrement l'état de tension apparent des apprentis, qu'il justifie à l'aide de prises d'informations dans l'environnement : «ils sont un petit peu stressés vu qu'ils ont un temps qui est donné» (1.1), «donc là on voit qu'il sont sous pression parce qu'il y a des pièces qui tombent/ des positions qui sont pas justes/» (1.6). Ce sont également des erreurs dans le positionnement du corps lors de l'activité de limage qui sont relevées : «alors on peut déjà voir déjà certains qui: qui: qui sont pas corrects/ qui sont pas en bonne position» (1.3). Enfin, ce sont des attitudes générales des apprentis à l'égard de la tâche qui font l'objet de commentaires : «puis en fait y a pas de réflexion avant de commencer la piècel qu'est-ce qu'il faut que je fasse/ pourquoi comment/», «ils foncent dessus sans se poser la question» (1.8).

Cette séquence d'interaction se compose également de nombreux énoncés à caractère anticipatoire, dans lesquels le formateur fait des prédictions sur des états à venir de l'activité en cours d'accomplissement. Ces prédictions portent tantôt sur des propriétés générales du déroulement des activités collectives («alors là aujourd'hui au fait ils vont faire beaucoup d'erreurs» [1.1]), tantôt sur des spécificités de certains apprentis («puis on verra certains d'ici cinq minutes ils vont se dire ah/ non il faut que je modifie il faut que je fasse comme ça comme ça comme çal» [1.9]; «puis d'autres qui vont y voir quand ce sera trop tard\» [1.10]). C'est ainsi une forme de différenciation entre certaines catégories d'apprentis qui semble ici mise en œuvre, et qui anticipe des trajectoires variables dans la conduite de la tâche.

Ces éléments de différenciation entre apprentis trouvent des formes d'expression plus précises dans la suite des échanges entre le formateur 
et le chercheur. La séquence retranscrite ci-dessous intervient environ une heure après la précédente. Les quatre apprentis présents dans l'atelier (Terry, Antoine, Brian, Daniel) ont avancé dans le limage de leur pièce métallique, mais des différences dans le rythme de travail commencent à se manifester.

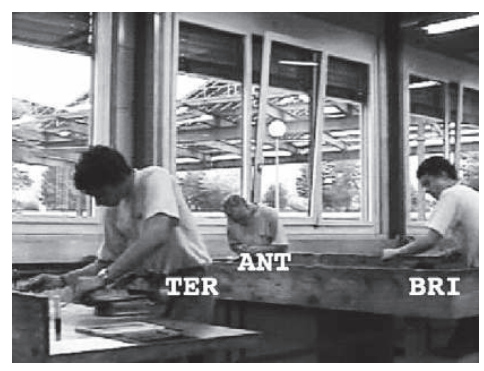

Image 3: Disposition des apprentis dans l'atelier.

Extrait 2. - Pièce métallique (Film nº 11, 13'25-14'35).

13'25 1. CHE : ((CHE s'approche de FOR, qui observe les apprentis au travail))

2. ils sont dans les temps/

3. FOR : ouais ouais non ils vont bien $\backslash$. après faudra voir si c'est plat mais $\backslash$. dans la vitesse XXX

13'50 4. CHE : y a pas trop trop de différences de/ de timing ils sont assez euh

5. FOR : ils sont assez en même temps ouais ouais $\backslash$. y a Terry on voit qu'il cherche beaucoup plus la petite bête que Brian ou Daniel/ . donc Antoine je me fais pas trop de soucis parce qu'il est un petit peu plus rapide/ . et en fait Antoine tire Brian/ . Terry lui il est tout seul je dirais il joue tout seul/ et puis en fait Daniel il regarde un petit peu ce qui se passe derrière pour rester un peu dans les temps $\backslash$..

14’30 6. CHE : après ils vont commencer à:

7. FOR : d'ici vingt minutes ils vont commencer à tracer à pointer/ . je pense qu'Antoine il va commencer à percer d'ici trois quarts d'heurel

Dans l'extrait 2, c'est désormais le chercheur qui sollicite le formateur et qui l'interroge d'abord sur le rythme de progression des apprentis («ils sont dans les temps/» [1.2]), puis sur les différences perceptibles entre ceux-ci ( «y a pas trop de différence de/ de timing ils sont assez euh» [1.4]). 
Cette nouvelle séquence d'interaction permet au formateur de rendre explicites des éléments de différenciation qu'il perçoit entre les apprentis. Ces éléments de différenciation prennent la forme de catégorisations successives, dans lesquelles les apprentis sont regroupés selon plusieurs caractéristiques. D'abord le degré de précision dans la conduite de l'activité de limage : «y a Terry on voit qu'il cherche beaucoup plus la petite bête que Brian ou Daniel» (1.5). Ensuite les variations rythmiques dans la progression de la tâche : «donc Antoine je me fais pas trop de soucis parce qu'il est un petit peu plus rapide/» (1.5). Enfin des logiques d'influences qui s'exercent réciproquement entre des sous-groupes d'apprentis : «et en fait Antoine tire Brian/ et Terry lui il est tout seul je dirais il joue tout seul/ et puis en fait Daniel il regarde un petit peu ce qui se passe derrière pour rester un peu dans les temps\» (1.5). On notera à ce propos que l'agencement de l'espace et la disposition des apprentis dans l'atelier ne sont pas sans influences sur cette catégorisation. Comme le montre bien l'illustration 3 ci-dessus, Brian et Antoine travaillent côte à côte et partagent un même espace visuel, alors que Terry et Daniel occupent des établis distincts et d'où l'activité des autres apprentis n'est pas immédiatement accessible visuellement. Ce sont ainsi d'une certaine manière les effets de cette écologie spatiale qui sont rendus manifestes dans le discours du formateur.

En résumé, on voit dans les deux séquences présentées ci-dessus comment les formes situées de l'explicitation de l'action ne thématisent pas seulement les dimensions techniques de l'activité en cours d'accomplissement, mais aussi ses dimensions proprement formatives. Ce sont en effet moins les gestes professionnels en tant que tels qui font l'objet de commentaires que l'activité des apprentis telle qu'elle se déploie dans un milieu configuré par le formateur à des fins d'apprentissage. À ce titre, les discours échangés avec le chercheur ne témoignent plus seulement d'une expertise professionnelle technique; ils mettent en œuvre une capacité réflexive, et partant une identité discursive, qui porte sur les dimensions didactiques de l'activité : la mise à disposition d'une consigne, la fixation de contraintes temporelles, la capacité de diagnostiquer des erreurs et d'interpréter des attitudes chez les apprentis, la catégorisation des apprentis selon leurs spécificités, l'anticipation des états futurs de l'activité et de la durée des étapes qui la constituent. 


\section{Potentialités et limites de l'explicitation située comme discours professionnel}

L'analyse de ces productions discursives permet de souligner à la fois la complexité et l'hybridité des verbalisations produites par les formateurs dans ces contextes d'interaction particuliers.

Dès lors qu'elles portent fréquemment sur les activités en cours d'accomplissement et qu'elles en proposent des formes interprétatives spécifiques, ces productions discursives mobilisent d'abord des aptitudes réflexives de la part des formateurs et configurent des espaces de parole dans lesquels la pratique devient un objet de pensée signifiant pour eux. Dans ce sens, ces verbalisations semblent bien réaliser des formes d'élaboration de l'action ou du moins des explicitations de l'expérience vécue, que celle-ci porte sur la pratique du métier (les gestes professionnels, l'organisation processuelle d'une technique, etc.) ou plus spécifiquement sur le rôle de formateur endossé en contexte par ces travailleurs (l'évaluation des conduites des apprentis, le repérage et l'anticipation des difficultés d'apprentissage, etc.). Mais d'un autre point de vue, ces espaces d'élaboration de l'expérience ne sont pas coupés des contextes d'action dont ils précisent la signification. Ils sont au contraire fortement «situés » dans les environnements à la fois pratiques et matériels dans lesquels ils se déploient. Cette accessibilité des ressources propres à l'accomplissement pratique de l'action génère d'abord des conduites spécifiques dans les interactions observées, comme par exemple la référence à des arrangements spatiaux mutuellement partagés (ex. la disposition des apprentis dans l'atelier). Elle implique également des logiques de synchronisation avec la conduite des activités réalisées, dont la nature et les conditions de déploiement peuvent favoriser ou au contraire inhiber des espaces potentiels d'explicitation. Dans les extraits présentés, c'est par exemple la nature même de la tâche proposée aux apprentis (i.e. la réalisation d'une pièce métallique de manière autonome et sans assistance) qui renforce la disponibilité du formateur pour des échanges avec le chercheur.

Nous proposons donc de désigner ces formes hybrides de verbalisation de l'agir comme des «explicitations situées», de sorte à faire ressortir les deux ingrédients majeurs qui les constituent. Ces formes situées de l'explicitation procèdent d'une double contextualisation, dès lors qu'elles font référence à la fois à un engagement du formateur en direction des apprentis et à une orientation en direction du chercheurobservateur. 
Cette double logique d'organisation du discours qui sous-tend les formes situées de l'explicitation permet à nos yeux de dépasser les logiques d'opposition parfois tranchées qui caractérisent différentes approches possibles des productions discursives dans leur rapport aux savoirs professionnels. Pour les tenants de la perspective «située» d'orientation ethnométhodologique par exemple, la posture «naturaliste» de l'observation vise à reconstituer la signification des conduites à partir d'un point de vue endogène et dynamique, dans lequel les acteurs se signalent mutuellement la manière dont ils s'orientent dans l'activité collective (Goodwin, 2000). Dans cette perspective, les verbalisations échangées constituent des ressources de cette coordination située et les savoirs professionnels s'y trouvent incorporés en tant qu'ils sont rendus publiquement interprétables par les participants à l'interaction. Dans cette perspective également, la conduite d'entretiens rétrospectifs ne constitue ni une nécessité ni même une condition suffisante pour interpréter les pratiques professionnelles. Au contraire, elle revient à mettre en place une activité à part entière, dont les liens de continuité avec la première sont difficiles à établir, et dont les enjeux et les modes de rationalité obéissent à des logiques propres (Mondada, 2005, p. 26). Pour les représentants du courant francophone d'analyse de l'activité d'orientation ergonomique en revanche, l'activité de travail et les savoirs qui lui sont liés ne sont pas interprétables en-dehors des verbalisations à postériori proposées par les acteurs eux-mêmes (Clot, 1999; Saujat, 2009). Theureau (2004, p. 15) par exemple souligne les paradoxes constitutifs des thèses ethnométhodologiques, qui postulent l'accès par l'observateur à des réalités qui échapperaient à la rationalité des acteurs eux-mêmes. Et il développe, dans le cadre de la méthode du «cours d'action», une technique d'entretien visant à reconstituer des formes de verbalisation à la fois distinctes de l'effectuation de l'action, mais présentant elles-mêmes un caractère situé et incorporé.

La sollicitation et l'analyse de formes situées de l'explicitation ne prétendent pas se substituer à l'une ou l'autre des positions en présence. Elles visent au contraire à souligner que des formes hybrides existent à l'intersection de ces approches, et que des discours professionnels peuvent être produits à la fois en action et à propos de celle-ci. Plus particulièrement, la démarche adoptée ici vise à montrer que la «mentalité analytique», propre aux approches interactionnelles, peut s'appliquer non plus seulement aux interactions didactiques elles-mêmes (Guernier, Durand-Guerrier et Sautot, 2006), mais également aux échanges spontanés ou provoqués impliquant le chercheur dans le cours de l'acti- 
vité d'observation. Dans cette perspective, résolument interactionnelle, la démarche d'explicitation est elle-même vue comme un accomplissement pratique procédant d'une logique à la fois dynamique et collective. Comme le montrent les données étudiées, la mise en visibilité des savoirs d'action par les formateurs résulte d'une construction progressive, façonnée par le processus interactionnel lui-même et son ordonnancement séquentiel. Le chercheur n'est pas absent de cette élaboration. Il lui arrive de l'initier, de l'encourager, ou encore de la reproduire, comme par exemple dans les extraits 1 et 2, dans lesquels des catégories initialement proposées par le formateur («ils sont un petit peu stressés vu qu'ils ont un temps qui est donné» [extrait 1,1.1]) sont ensuite reprises par le chercheur à l'occasion d'un état ultérieur d'avancement de la tâche («ils sont dans les temps/» [extrait 2,1.1]). C'est en ceci que les formes situées de l'explicitation gagnent à être envisagées non plus seulement comme des contenus verbalisés, mais aussi comme des processus d'accomplissement dynamiques qui rendent ces contenus accessibles à l'analyse.

Dans cet article, nous avons porté notre attention sur des discours professionnels qui se déploient à la marge des démarches de recherche classiquement adoptées dans le champ des sciences du travail et de la formation. Au terme de ce parcours, il importe d'en questionner à la fois les potentialités et les limites. Les formes situées de l'explicitation de l'action relèvent-elles d'un écueil méthodologique dans lequel il faudrait éviter de tomber ou constituent-elles au contraire une ressource qu'il n'est pas inintéressant d'explorer, voire de reproduire, dans la recherche en formation professionnelle?

D'un point de vue méthodologique, l'hybridité manifeste dont relève l'explicitation située n'est pas sans dangers. Pour les tenants de la perspective ethnométhodologique conversationnelle, elle constitue sans doute un résidu manifeste d'une forme d'intrusion du chercheur dans les réalités qu'il observe et, partant, une concession de taille aux exigences d'une démarche «naturaliste» dans laquelle l'observateur cherche à minimiser autant que possible ses effets sur la situation. À l'inverse, pour les tenants de démarches plus cliniques ou sémiologiques en analyse de l'activité, les contingences propres à la réalisation du travail n'offrent pas les conditions favorables à la verbalisation, les temporalités de l'effectuation de l'activité étant peu propices aux élaborations rétrospectives. Dans cette mouvance, les techniques d'entretien classiquement employées dans ce champ (l'explicitation, l'instruction au sosie, l'auto-confrontation simple ou croisée) visent précisément un 
dépassement de ces limites et la mise en place de conditions qui permettent véritablement le développement de formes élaborées de telles explicitations. Ainsi donc, l'émergence de formes situées d'explicitations relèverait-elle davantage d'un métissage inclassable, voire d'un éclectisme méthodologique mal maitrisé.

Pourtant, selon une logique alternative, les sortes de réalités empiriques que nous avons relevées et proposé d'étudier ici ne semblent pas sans intérêt dans le cadre d'une approche discursive des processus de professionnalisation. Et ce, pour deux raisons au moins, qui méritent d'être mentionnées.

La première raison a trait aux possibilités qu'offrent les formes situées d'explicitation de mettre en visibilité la diversité et la complexité des savoirs professionnels qui sous-tendent les pratiques d'enseignement et de formation et, plus généralement, les rapports que les professionnels entretiennent avec ces savoirs. Dans une publication récente, Vanhulle (2009) identifie par exemple quatre catégories de savoirs en lien avec la professionnalisation des enseignants dans des dispositifs de formation : a) les savoirs académiques, qui se rapportent aux domaines scientifiques de référence et qui permettent de fonder la profession; b) les savoirs institutionnels, correspondant aux attentes sociales et aux réalités organisationnelles de l'enseignement; c) les savoirs de la pratique, mis à disposition par les membres experts de la profession; d) et enfin les savoirs expérientiels, que les acteurs se forgent dans leur parcours biographique propre. Or, il n'est pas inintéressant d'observer qu'en dépit du contexte éducatif singulier qui nous concerne ici, ce sont bien des savoirs en lien avec une pluralité de ces catégories qui semblent mis en circulation dans les discours produits par le formateur. Comme nous l'avons esquissé ici, ces verbalisations portent fréquemment sur les savoirs de référence en lien avec une pratique professionnelle (ex. comment mettre les longueurs d'une pièce à l'équerre). À d'autres moments, elles portent davantage sur les dimensions proprement didactiques de l'activité telles qu'elles se rapportent aux enjeux institutionnels liés à l'organisation d'un centre de formation (ex. le choix de la tâche et la nature des consignes mises à disposition). Enfin, ces verbalisations ne sont pas sans rapports avec l'expérience pratique accumulée par le formateur, et qui conduit ce dernier à diagnostiquer certaines difficultés d'apprentissage ou à anticiper des rythmes de progression dans l'activité des apprentis. C'est donc en ce qu'elles rendent à certains égards accessibles des savoirs à la fois techniques, institutionnels, pratiques et expérientiels que les formes 
de verbalisation étudiées ici nous semblent constituer, au-delà de leur hybridité constitutive, une réelle valeur heuristique pour la recherche en formation professionnelle.

La deuxième raison pour laquelle l'étude des formes situées de l'explicitation semble prometteuse réside dans la possibilité qu'elle offre de verbaliser l'expérience dans un cadre communicationnel certes influencé par la présence du chercheur, mais qui ne se coupe pas des conditions réelles d'effectuation du travail. Dans les données constituées et analysées ici, le chercheur endosse en effet une posture bien particulière. Il se positionne comme une instance d'observation qui ne se dissout ni dans le point de vue radicalement endogène des membres, ni dans celui d'une expertise exogène, visant à prescrire ou évaluer les pratiques attestées au nom de savoirs issus de la recherche. Cette posture nous parait particulièrement propice à la sollicitation et à l'accompagnement d'une démarche réflexive, dès lors qu'elle invite les formateurs à rendre leur activité interprétable dans un contexte à la fois distinct de leur environnement de travail habituel, mais qui en constitue une forme d'expérience proche. Cette proximité n'est sans doute pas étrangère au caractère souvent spontané des démarches réflexives observées. Elle pourrait contribuer à dépasser ce qui constitue un écueil maintes fois relevé dans les démarches d'analyse de l'activité d'inspiration ergonomique à propos des professions techniques (Boutet, 1995) : la difficile verbalisation du travail et l'absence de mots et de genres de discours constitués pour dessiner dans ses subtiles nuances les contours de l'expérience professionnelle. Dans cette perspective, l'aménagement de tels espaces discursifs peut être vu comme une opportunité de développement professionnel des formateurs, et une occasion pour eux d'endosser des identités complexes, à l'intersection d'une compétence technique et d'une compétence didactique. Ces espaces discursifs sont certes difficilement transposables à l'ensemble des contextes éducatifs, mais ils nous semblent constituer un outil particulièrement adapté aux réalités de l'activité des formateurs de la formation professionnelle initiale.

\section{RÉFÉRENCES BIBLIOGRAPHIQUES}

Boutet J. (1995): «Le travail et son dire», dans J. Boutet (dir.), Paroles au travail, Paris, L'Harmattan, p. 247-267.

CLOT Y. (1999) : La fonction psychologique du travail, Paris, Presses universitaires de France. 
DuBs R. (2006) : Rapport d'expertise sur les questions concernant la formation professionnelle en Suisse, Berne, HEP Verlag.

Filliettaz L., de Saint-Georges I. et Duc B (2008) : "Vos mains sont intelligentes! » : Interactions en formation professionnelle initiale, Université de Genève, Cahiers de la section des sciences de l'éducation, $\mathrm{n}^{\mathrm{o}} 117$.

- (2009) : «Interactions et dynamiques de participation en formation professionnelle initiale», dans M. Durand et L. Filliettaz (dir.), Travail et formation des adultes, Paris, Presses universitaires de France, p. 95-124.

- (2010) : «Reformulation, re-sémiotisation et trajectoires d'apprentissage en formation professionnelle initiale : le cas de l'enseignement du giclage du mortier en maçonnerie», dans A. Rabatel (dir.), Les reformulations plurisémiotiques en contexte de formation, Besançon, Presses universitaires de Franche-Comté, p. 255-277.

Goodwin C. (2000) : «Action and embodiment within situated human interaction », Journal of Pragmatics, $\mathrm{n}^{\circ}$ 32, p. 1489-1522.

Guernier M.-C., Durand-Guerrier V. et Sautot J.-P. (dir.) (2006) : Interactions verbales, didactiques et apprentissages, Besançon, Presses universitaires de Franche-Comté.

Marcel J.-F., Olry P., Rauthier-Bautzer E. et Sonntag M. (2002) : «Les pratiques comme objet d'analyse», Revue française de pédagogie, $\mathrm{n}^{\circ} 138$, p. 135-170.

Mondada L. (2005) : Chercheurs en interaction : comment émergent les savoirs, Lausanne, Presses polytechniques et universitaires romandes.

Plazaola Giger I. et Stroumza K. (dir.) (2007) : Paroles de praticiens et description de l'activité. Problématisation méthodologique pour la formation et la recherche, Bruxelles, De Boeck.

Relieu M., Salembier P. et Theureau J. (dir.) (2004) : @ ctivité, vol. 1, nº 2, Activité et action / cognition située [en ligne]. Disponible sur <http:// www.activites.org/> [consulté le 24 juin 2010].

Saujat F. (2009) : «L'analyse du travail comme source et ressource de formation : le cas de l'orientation en collège», dans M. Durand et L. Filliettaz (dir.), Travail et formation des adultes, Paris, Presses universitaires de France, p. 245-274.

Theureau J. (2004) : «L'hypothèse de la cognition (ou action) située et la tradition d'analyse du travail de l'ergonomie de langue française» [en ligne], @ctivité, vol. 1, n 2, Activité et action / cognition située, p. 11-25. Disponible sur <http://www.activites.org/v1n2/theureau.pdf> [consulté le 24 juin 2010].

VANHUlLE S. (2009) : «Savoirs professionnels et construction sociodiscursive de l'agir», Bulletin suisse de linguistique appliquée, $\mathrm{n}^{\circ} 90, \mathrm{p} .167-$ 188 . 


\section{ANNEXE}

\section{Conventions de transcription}

$\begin{array}{ll}\mid & \begin{array}{l}\text { intonation montante } \\ \text { intonation descendante }\end{array} \\ \mathrm{XX} & \text { segments intranscriptibles } \\ \ldots \ldots & \text { pauses de durée variable } \\ <\mathrm{mhmm}> & \begin{array}{l}\text { régulateurs verbaux } \\ ((\text { commentaire })) \\ \end{array} \\ & \begin{array}{l}\text { commentaire du transcripteur, relatif à des conduites } \\ \text { gestuelles ou des actions non verbales }\end{array}\end{array}$

